\title{
Global Performance of Covariant Density Functional Theory in Description of Charge Radii and Related Indicators
}

\author{
A.V. Afanasjev ${ }^{1}$, U.C. Perera ${ }^{1}$, P. Ring ${ }^{2}$ \\ ${ }^{1}$ Department of Physics and Astronomy, Mississippi State University, \\ MS 39762, USA \\ ${ }^{2}$ Fakultät für Physik, Technische Universität München, \\ D-85748 Garching, Germany
}

Received 30 October 2021

doi: https://doi.org/10.55318/bgjp.2021.48.5-6.375

\begin{abstract}
A short review of existing efforts to understand charge radii and related indicators on a global scale within the covariant density functional theory (CDFT) is presented. Using major classes of covariant energy density functionals (CEDFs), the global accuracy of the description of experimental absolute and differential charge radii within the CDFT framework has been established. This assessment is supplemented by an evaluation of theoretical statistical and systematic uncertainties in the description of charge radii. New results on the accuracy of the description of differential charge radii in deformed actinides and light superheavy nuclei are presented and the role of octupole deformation in their reproduction is evaluated. Novel mechanisms leading to odd-even staggering in charge radii are discussed. Finally, we analyze the role of self-consistency effects in an accurate description of differential charge radii.
\end{abstract}

KEY WORDS: Covariant density functional theory, charge radii, differential charge radii, theoretical uncertainties, self-consistency effects.

\section{Introduction}

The charge radii are among the most fundamental properties of atomic nuclei. Essential information on the saturation density of symmetric nuclear matter is imprinted into them. They also depend on the properties of nuclear forces and nuclear many-body dynamics. Charge radii have been in the focus of experimental and theoretical studies for a long time and, in the last decade, there was a significant increase in the interest to them due to the availability of new facilities and new techniques. Absolute charge radii are determined via the studies of 
muonic spectra and electronic scattering experiments $[1,2]$. However, such experiments are impossible in radioactive elements. Laser spectroscopy is a more flexible tool in that respect and allows a significantly higher precision of the measurements of the changes of charge radii within the isotopic chain [1,2].

The CDFT [3] is one of the modern theoretical tools which has been used with reasonable success to the description of charge radii and related indicators. For example, the first-ever successful description of the kink in charge radii of the lead isotopes has been achieved in the CDFT framework in Ref. [4] and in the 90 ies it followed by a study of differential charge radii in spherical even-even nuclei of the $\mathrm{Ca}, \mathrm{Sn}$, and $\mathrm{Pb}$ isotopic chains in Ref. [5]. However, only during the last seven years did new CDFT results appear, dedicated to the study of charge radii, related indicators, and other connected issues. The goal of this paper is to present a short review of different facets of physics of charge radii in CDFT and to provide new clarifying results. All the quoted and new calculations are performed within the framework of relativistic Hartree-Bogolibov (RHB) approach with separable pairing interaction of Ref. [6]. The technical details of the RHB approach and numerical details of the calculations are presented in Ref. [7]. The calculations are carried out with the CEDFs (such as NL3* [8], DD-ME2 [9], DD-ME $\delta$ [10], DD-PC1 [11], PC-PK1 [12] and NL5(C), NL5(D) and NL5(E) [13]) representing the major classes of the CDFT models.

\section{Physical Observables}

We focus here on physical observables, which provide information on charge radii and related indicators. The charge radii were calculated from the corresponding point proton radii as (see Ref. [7])

$$
r_{c h}=\sqrt{\left\langle r^{2}\right\rangle_{p}+0.64} \mathrm{fm},
$$

where $\left\langle r^{2}\right\rangle_{p}$ stands for proton mean-square point radius and the factor 0.64 accounts for the finite-size effects of the proton.

In addition, two differential indicators are commonly used to facilitate the quantitative comparison of the experimental results with those from theoretical calculations. One of them is the differential mean-square charge radius

$$
\delta\left\langle r^{2}\right\rangle_{p}^{N, N^{\prime}}=r_{\mathrm{ch}}^{2}(N)-r_{\mathrm{ch}}^{2}\left(N^{\prime}\right),
$$

where $N^{\prime}$ is the neutron number of the reference nucleus. Another is the threepoint indicator

$$
\Delta\left\langle r^{2}\right\rangle^{(3)}(N)=\frac{1}{2}\left[r_{\mathrm{ch}}^{2}(N-1)+r_{\mathrm{ch}}^{2}(N+1)-2 r_{\mathrm{ch}}^{2}(N)\right],
$$

which quantifies OES in charge radii. 


\section{Global Performance of the CDFT in the Description of Charge Radii}

The global performance of CDFT in the description of charge radii for major classes of CEDFs has been studied in Refs. [7, 13, 14], and Figure 1 presents an example of a comparison between theory and experiment. The following rms deviations $\Delta r_{\mathrm{ch}}^{\mathrm{rms}}$ between calculated and experimental charge radii have been reported: $\Delta r_{\mathrm{ch}}^{\mathrm{rms}}=0.0283 \mathrm{fm}$ for CEDF NL3 $*, \Delta r_{\mathrm{ch}}^{\mathrm{rms}}=0.0230 \mathrm{fm}$ for DD-ME2, $\Delta r_{\mathrm{ch}}^{\mathrm{rms}}=0.0329 \mathrm{fm}$ for DD-ME $\delta^{*}, \Delta r_{\mathrm{ch}}^{\mathrm{rms}}=0.0253 \mathrm{fm}$ for DDPC1, $\Delta r_{\mathrm{ch}}^{\mathrm{rms}}=0.0284 \mathrm{fm}$ for NL5(C), $\Delta r_{\mathrm{ch}}^{\mathrm{rms}}=0.0277 \mathrm{fm}$ for NL5(D), and $\Delta r_{\mathrm{ch}}^{\mathrm{rms}}=0.0288 \mathrm{fm}$ for NL5(E). Note that the DD-PC1 functional has been adjusted only to nuclear binding energies while other functionals include the information on charge radii in their fitting protocols. The average rms deviations of calculated $r_{\mathrm{ch}}$ from experimental ones for this group of functionals are at the level of $0.028 \mathrm{fm}$. Considering that the average experimental rms charge radius in the nuclear chart is around $4.8 \mathrm{fm}$ (see, for example, Figure 23 in Ref. [7] and Figures 2-4 in Ref. [1]), this amounts to a high average precision of $0.58 \%$ in the prediction of charge radii.

Figure 1 reveals several regions on the nuclear chart which exhibit enhanced (with respect of average trend) differences $\left(r_{\mathrm{ch}}^{\mathrm{exp}}-r_{\mathrm{ch}}^{\mathrm{th}}\right)$ between experimental and calculated charge radii. These are (i) proton-rich nuclei around $\operatorname{Hg}(Z=80)$, (ii) proton-rich nuclei around $\operatorname{Sr}(Z=38)$ located below the $N=50$ shell closure

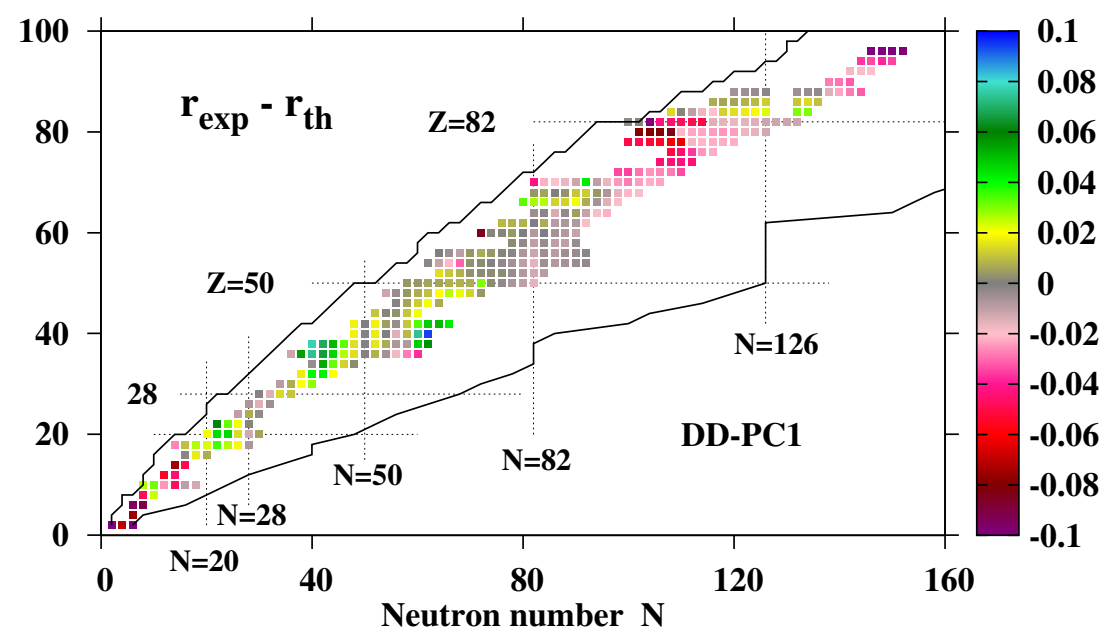

Figure 1. (Color online) The difference between measured and calculated charge radii $r_{\mathrm{ch}}$ for the CEDF DD-PC1. Two-proton and two-neutron drip lines obtained with this functional are shown by solid black lines. The experimental data are taken from Ref. [1]. Based on figure from Ref. [14]. 
and (iii) light and very light nuclei in the region with $Z<20$. The detailed analysis of the nuclei in the regions (i) and (ii) performed in Refs. [15-19] clearly indicates that shape coexistence and the correlations beyond mean-field are the sources of these discrepancies. Note that the latter are neglected in our studies. The importance of the correlations beyond mean-field is also expected to be increased in light and very light nuclei, the potential energy surfaces of which are very frequently soft in collective coordinates. The fact that the accuracy of the description of the masses declines with decreasing mass also points in that direction (see discussion in Sec. V of Ref. [7]). Thus, the inclusion of the correlations beyond mean-field in the regions (i-iii) may improve the description of charge radii and binding energies. The accuracy of the description of charge radii also deteriorates in the actinides (see Figure 1). However, this is most likely due to the extrapolations used in Ref. [1] for the definition of absolute charge radii since they have not been measured experimentally in the $Z>83$ nuclei (uranium is an exception).

\section{Statistical and Systematic Uncertainties in the Description of Charge Radii}

Because of underlying physical approximations in modeling of the nuclear many-body problem it is important to estimate statistical and systematic theoretical uncertainties in the predictions of charge radii. This is especially important when one deals with the extrapolations beyond the known regions, as, for example, in particle number or deformation, since experimental data which act as a substitute of an exact solution are not available there. Such estimates are also required for the evaluation of the predictive power of the models and the robustness of their predictions.

Systematic theoretical uncertainties emerge from underlying theoretical approximations about the form of the energy density functional. In the DFT framework, there are two major sources of these approximations, namely, the range of interaction and the form of the density dependence of the effective interaction $[3,20,21]$. In reality, only the lower limit of these uncertainties is possible to estimate (see Refs. [7, 13, 22]) and even then, it contains a degree of arbitrariness related to the choice of the set of the functionals. In our works, it is estimated via the spread of theoretical predictions within a set of selected functionals (NL3*, DD-ME2, DD-PC1, DD-ME $\delta$ ) as

$$
\Delta r_{\mathrm{ch}}(Z, N)=\left|r_{\mathrm{ch}}^{\max }(Z, N)-r_{\mathrm{ch}}^{\min }(Z, N)\right|,
$$

where $r_{\mathrm{ch}}^{\max }(Z, N)$ and $r_{\mathrm{ch}}^{\min }(Z, N)$ are the largest and smallest values of the charge radii obtained with the four employed CEDFs for the $(Z, N)$ nucleus [7]. The charge radii spreads are shown in Figure 2. For the nuclei shown in Figure 1 they are on the level of $0.01 \mathrm{fm}$ for the majority of medium mass and heavy nuclei but become larger for $Z \leq 40$ nuclei where they exceed $0.02 \mathrm{fm}$ 


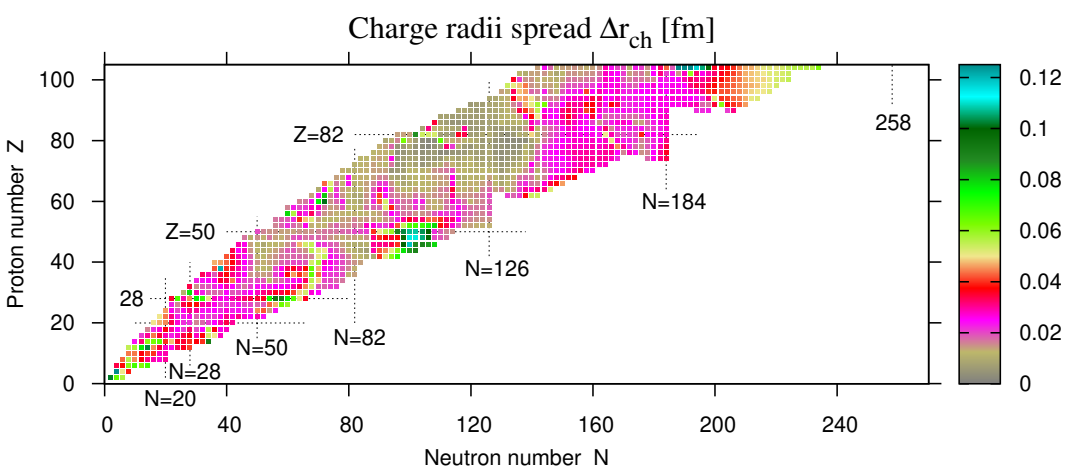

Figure 2. (Color online) Systematic uncertainties in the description of charge radii. From Ref. [7].

in most of the nuclei. The latter feature is most likely due to softer potential energy surfaces of such nuclei and thus stronger dependence of the results on the details of the underlying single-particle structure. As a result, in some nuclei the systematic theoretical uncertainties are either comparable or larger than the differences between experimental and calculated charge radii (compare Figures 2 and 1). This fact has to be kept in mind when performing a detailed comparison between theory and experiment.

Statistical errors $\sigma\left(r_{\mathrm{ch}}\right)$, which apply only to a given functional, are due to the details of the fitting protocol, such as the choice of experimental data and the selection of adopted errors (see Refs. [13,22]). They are shown in Figure 3 for the NL5(C) functional for several isotopic chains of spherical nuclei. However, similar magnitude of $\sigma\left(r_{\mathrm{ch}}\right)$ is expected both for other functionals and for deformed nuclei [13]. One can see that, on average, they are by a factor of approximately 6 smaller than global rms deviations between theory and experiment. They are also substantially smaller than systematic theoretical uncertainties for charge radii. Note that the $\sigma\left(r_{\mathrm{ch}}\right)$ values show some fluctuations as a function of neutron number due to the underlying shell structure; they become especially pronounced in the Ni isotopes. These fluctuations are expected to wash out in deformed nuclei because of a more equal distribution of deformed single-particle states originating from different spherical subshells. On average, the $\sigma\left(r_{\mathrm{ch}}\right)$ values shown in Figure 3 display a very modest increase on going from the twoproton to the two-neutron drip line. This is in contrast to the results obtained in the Skyrme DFT calculations with the UNEDF0 functional (see Ref. [23]) which for the nuclei near the proton drip line shows $\sigma\left(r_{\mathrm{ch}}\right)$ values comparable to those obtained in the CDFT(NL5(C)) calculations, but are by a factor of 17-33 larger at the neutron drip line (see Ref. [13]). Based on these considerations, one can conclude that statistical errors represent only a relatively small part of the total 


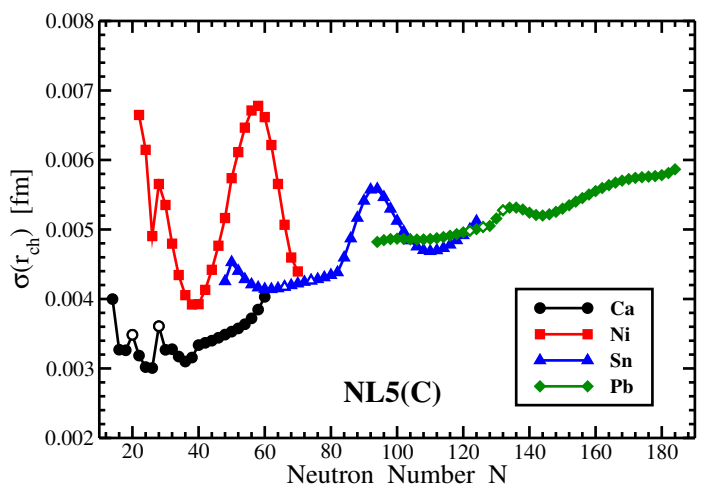

Figure 3. (Color online) The propagation of statistical errors in charge radii with neutron number for the $\mathrm{Ca}(Z=20)$, Ni $(Z=28)$, Sn $(Z=50)$, and $\mathrm{Pb}(Z=82)$ isotopic chains. The RHB calculations have been performed at spherical shape with the NL5(C) functional for all even-even nuclei located between the two-proton and two-neutron drip lines (see Ref. [13] for details). Open symbols are used to indicate the nuclei whose experimental data have been used in the fitting protocol of this functional. From Ref. [13].

theoretical error in the prediction of charge radii and in many cases they can be neglected.

\section{Differential Charge Radii: The Examples of the Sn/Gd Region and the Region of Actinides and Light Superheavy Nuclei}

The differential charge radius $\delta\left\langle r^{2}\right\rangle^{N, N^{\prime}}$ (see Eq. (2)) is an indicator which is very sensitive to the shell closures, underlying single-particle structure, pairing interaction, and occupation pattern of the single-particle states and their evolution with neutron number (see Refs. $[15,24]$ and references quoted therein). Their systematic global investigation has been performed within the CDFT framework in Ref. [15] and theoretical results were compared with experimental data which crosses the neutron shell closures at $N=28,50,82$, and 126. An example of such a comparison is presented in Figure 4. Absolute differential radii of different isotopic chains and their relative properties are reasonably well described in model calculations in the cases when the mean-field approximation is justified. The observed clusterization of differential charge radii of different isotopic chains (see Ref. [25]) is well described above the $N=50$ and $N=126$ shell closures (see Ref. [15]). However, a similar clusterization above the $N=28$ and $N=82$ shell closures is not that well reproduced due to deficiencies in the underlying single-particle structure.

In the present paper, we focus on differential charge radii of deformed actinides and light superheavy nuclei which were not covered in Ref. [15]. The aim is to understand the accuracy of the description of the $\delta\left\langle r^{2}\right\rangle^{N, N^{\prime}}$ values in high- 


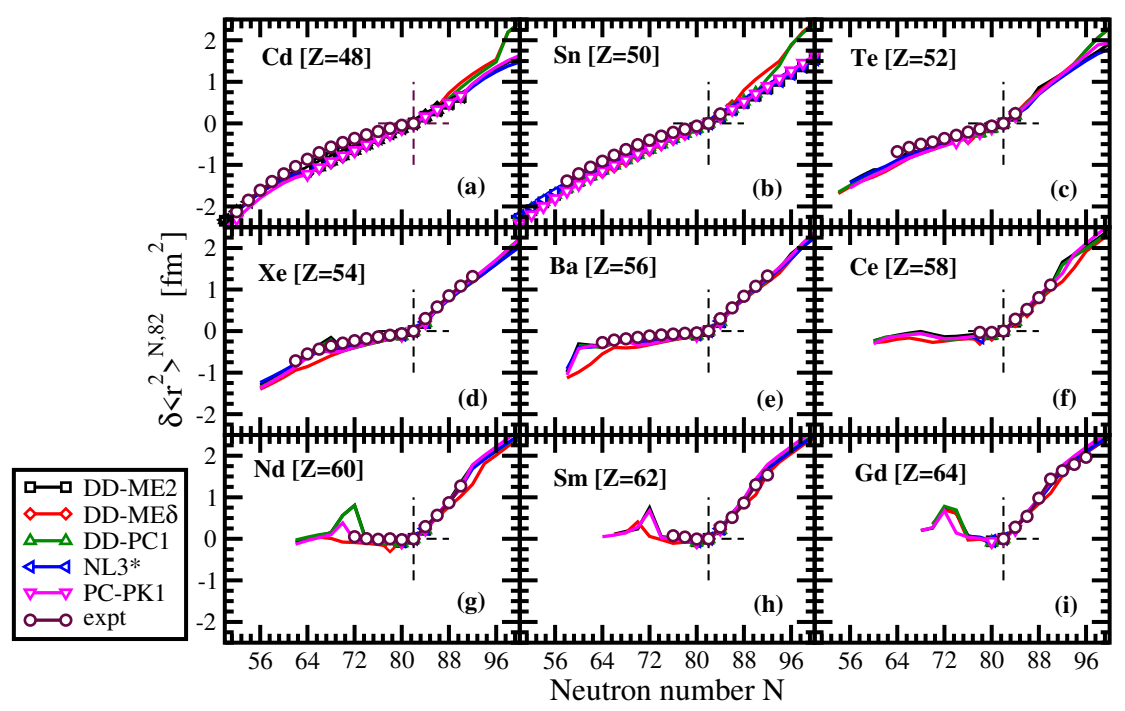

Figure 4. (Color online) The experimental and calculated differential charge radii $\delta\left\langle\left\langle r^{2}\right\rangle^{N, N^{\prime}}\right.$ of the indicated isotopic chains. The experimental data are taken from Ref. [1]. The calculations are performed with the indicated CEDFs. Theoretical results are shown by lines with symbols and by only lines for spherical and deformed ground states, respectively.

$Z$ systems and the impact of octupole deformation on differential charge radii. Note that due to the difficulties of the measurements of absolute charge radii in the $Z>83$ nuclei, the experimental data covers only differential charge radii [the only exception is uranium]. The experimental and calculated differential charge radii of the $\mathrm{Th}, \mathrm{U}, \mathrm{Pu}, \mathrm{Cm}$, and No isotopic chains are compared in Figure 5. Note that only even-even nuclei are considered in the calculations. The format of the figure is similar to that of Figure 2 of Ref. [28] in which the results of Skyrme DFT calculations with UNEDF1 and SV-min functionals are compared with experimental data. Note that the RHB calculations indicate the presence of octupole deformation in low- $N$ Th and $\mathrm{U}$ isotopes (see Ref. [26]) but, in a given nucleus, it somewhat depends on the employed CEDF and on the type of the DFT $[26,30]$. The accounting of octupole deformation leads to an increase of the $\delta\left\langle r^{2}\right\rangle^{N, N^{\prime}}$ value by approximately $\approx 0.1 \mathrm{fm}^{2}$ and leads to an improvement in the description of differential charge radii in the Th and $\mathrm{U}$ isotopes.

On average, the accuracy of the description of differential charge radii obtained with covariant (DD-ME2, DD-PC1, PC-PK1, and NL3*) and non-relativistic energy density functionals (UNEDF1 and SV-min) is similar (compare Figure 5 in the present paper with Figure 2 in Ref. [28]). Among the employed CEDFs, the best agreement with experiment is obtained for the Th isotopes by DD-ME2, 


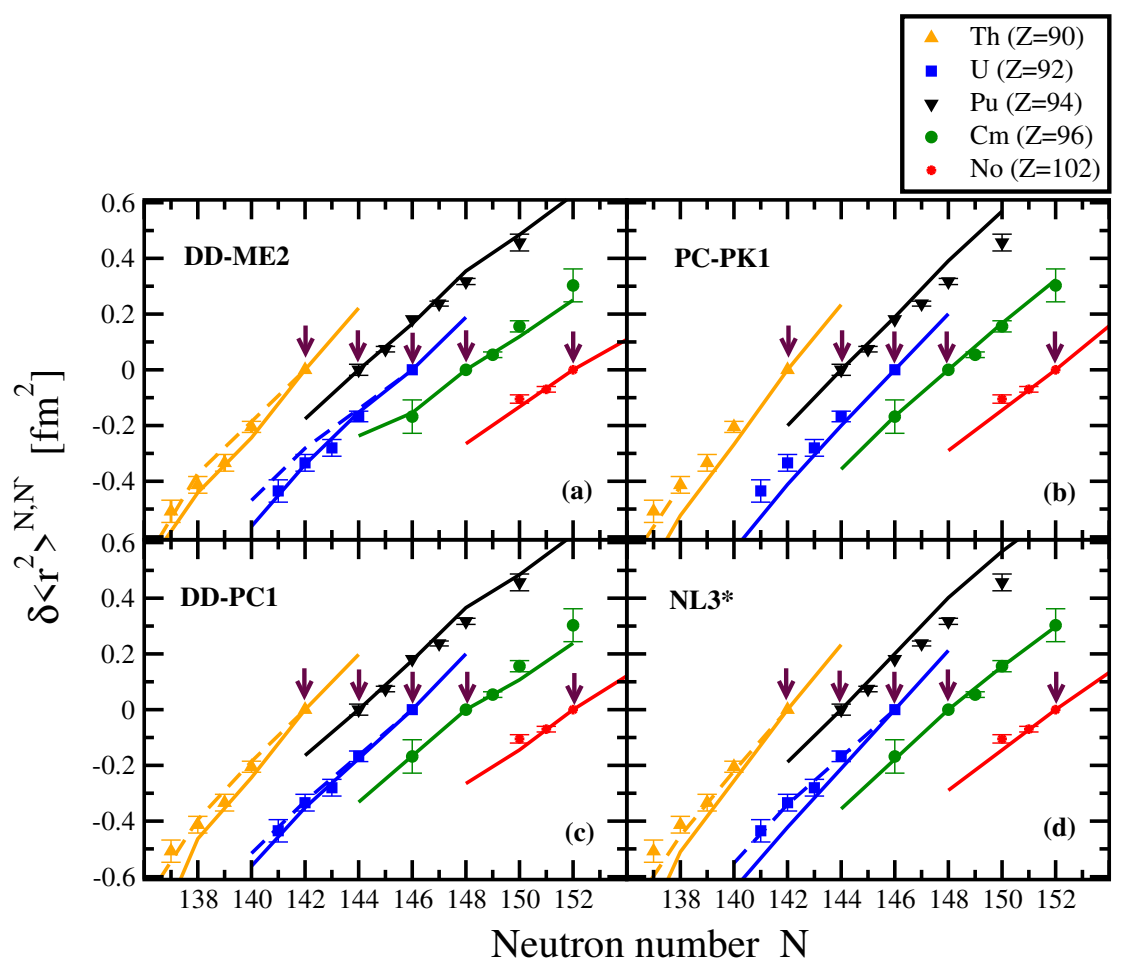

Figure 5. (Color online) The experimental and calculated differential charge radii $\delta\left\langle r^{2}\right\rangle^{N, N^{\prime}}$ of the indicated isotopic chains. The results of reflection symmetric and reflection asymmetric (with octupole deformation) RHB calculations are shown by solid and dashed lines, respectively. Note that the latter are based on the results obtained in Ref. [26] and are shown only for the nuclei which have non-zero octupole deformation $\beta_{3}$. The arrows indicate the reference nuclei in the isotopic chains. The experimental data are taken from Refs. $[1,27,28]$.

DD-PC1 and NL3*, for the U isotopes by DD-PC1 and NL3*, for the Pu isotopes by DD-ME2 and DD-PC1, and for the Cm isotopes by PC-PK1 and NL3* (see Figure 5). The results of the calculations for the No isotopes are the same with all employed functionals ${ }^{1}$. The local deviations between theory and experiment for $\delta\left\langle r^{2}\right\rangle^{N, N^{\prime}}$ are most likely due to the differences between experimental and calculated energies of the deformed quasiparticle states (see Refs. [31-33]) which leads to some dissimilarity of their contributions to charge radii. The same is true when the results of the calculations with two functionals are compared (see Sect. IV of Ref. [15] for a discussion of the impact of the underlying

\footnotetext{
${ }^{1}$ Note that the analysis of the No isotopes performed in Ref. [29] suggests that the measurements of at least two atomic transitions would allow to disentangle the contributions of the changes in deformation and charge radius into field isotopic shifts.
} 
single-particle structure and occupation pattern of the single-particle states on differential charge radii of the spherical $\mathrm{Pb}$ nuclei).

\section{New Mechanisms Leading to Odd-Even Staggering in Charge Radii}

The description of odd-even staggering (OES) in charge radii has been a challenge for a long time. In the most commonly accepted scenario, it is due to the gradient terms both in the surface part of the energy density functional and in the pairing interaction (see Refs. [34-36]). However, this has recently been challenged in Ref. [37] (see also Ref. [24] for a more detailed study) where it has been shown that the scattering of the occupation of different single-particle states between even-even and odd nuclei in the isotopic chain can lead to OES in charge radii. This scattering is due to the fact that particle-vibration coupling (PVC) leads to a change of the relative order of the single-particle states in odd$N$ nuclei as compared with even- $N$ ones in which PVC plays a minor role.

The analysis of Refs. [15, 24,37] also clearly indicates the importance of taking into account both the structure of the ground state in odd- $A$ nuclei and the fact that the DFT calculations do not reproduce the structure of the ground states in odd- $A$ nuclei in more than half of the cases (see Refs. [31, 32, 38, 39]). These facts have been ignored in the absolute majority of the studies of OES in charge radii. The importance of the accounting of these facts is illustrated in Figure 6 in which OES in charge radii of the $\mathrm{Sn}$ isotopes obtained with two procedures (LES and EGS) in the RHB calculations of odd- $N$ nuclei are compared. In the LES procedure, the lowest in energy calculated configuration is used. In the EGS procedure, the configuration with the spin and parity of the blocked state corresponding to those of the experimental ground state is employed, although it is not necessarily the lowest in energy. The results of the RHB calculations with the LES procedure significantly underestimate the magnitude of experimental OES and occasionally provide a wrong phase of the OES (see Figure 6(b)). The use of the EGS procedure significantly improves the description of both the phases and the magnitude of OES (see Figure 6(a)). However, even in that case, the magnitude of OES is underestimated on average by a factor of approximately two.

The remaining discrepancies between theory and experiment suggest that the energy considerations discussed above are not sufficient and special attention has to be paid to the wavefunction of the ground state in odd- $N$ nuclei. There is a substantial difference between the ground states in even-even and odd- $A$ nuclei with stiff parabola-like potential energy surfaces (such as the Sn isotopes). Indeed, the impact of the correlations beyond mean-field on the wavefunction of the ground state is relatively small in even-even nuclei, but it is substantial in odd- $A$ ones. The latter is due to the coupling of the single-particle motion with phonons (particle-vibration coupling) which leads to a substantial fragmenta- 


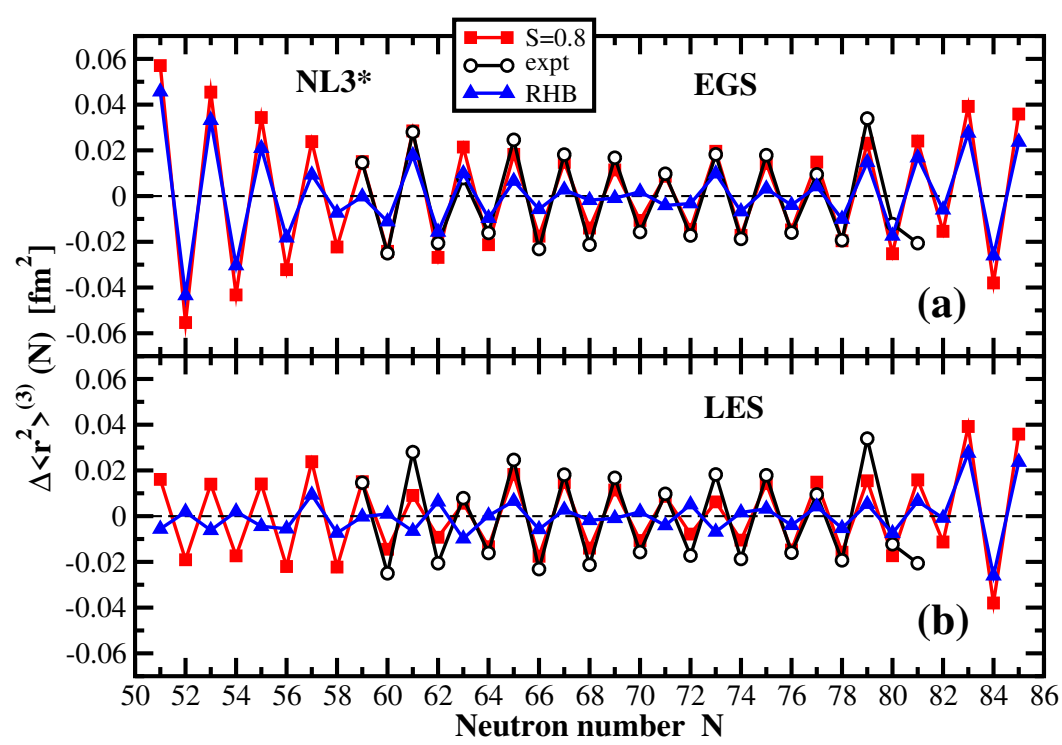

Figure 6. (Color online) OES in charge radii of the Sn isotopes. The experimental data are taken from Ref. [1]. The RHB results with the NL3* CEDF are shown by blue lines with triangles for the LES and EGS procedures in odd- $N$ nuclei. The results corrected for the fragmentation of the single-particle content of the dominant single-particle state in odd- $N$ nuclei within the schematic model discussed in the text are shown by red lines with squares for a representative spectroscopic factor $S=0.8$.

tion of the wavefunction; this feature is confirmed in numerous experiments (see Refs. [38-40] and references quoted therein). This reduces the single-particle content of the unpaired neutron ground state in odd- $A$ nuclei to the value characterized by the spectroscopic factor $S(S \leq 1.0)$. As a result, the pull provided by the odd neutron on the proton radii (see Refs. [15,41] and Section 7) is reduced as compared with the mean-field value. Assuming that at the mean-field level the increase of charge radii on going from even to odd neutron number in the even $Z$ isotopic chain is $\Delta\left(r_{\mathrm{ch}}\right)_{\mathrm{MF}}^{q p}=r_{\mathrm{ch}}(N+1)-r_{\mathrm{ch}}(N)$ [here $N$ is even], the accounting of the fragmentation of the wavefunction in odd- $N$ nuclei will reduce this increase to $\Delta\left(r_{\mathrm{ch}}\right)_{\text {frag }}=S \Delta\left(r_{\mathrm{ch}}\right)_{\mathrm{MF}}^{q p}$. Using this schematic model (see Ref. [15] for more details), one can show that the fragmentation of the structure of the unpaired neutron in odd- $N$ nuclei due to particle-vibration coupling leads to an increase of the magnitudes of OES in charge radii and correct phases of the OES both in the LES and EGS procedures for typical values of $S$ (see Figure 6). Both of these factors act in the direction of improving the agreement with experiment. 


\section{The Role of Self-Consistency Effects}

As reviewed in the introduction of Ref. [15], the absolute majority of the studies of differential charge radii has been performed either in DFT or in ab initio approaches. However, it is well known that these types of models have some deficiencies in the description of spectroscopic properties related to the energies of the single-particle states and their wave functions [31-33,39,42]. In contrast, the spherical shell models with empirical interactions provide a better description of experimental spectroscopic data in spherical nuclei located in the vicinity of doubly magic nuclei and microscopic+macroscopic (mic+mac) models based on phenomenological potentials such as the Woods-Saxon one do the same in the region of deformed nuclei. However, we are not aware of successful investigations of differential charge radii within these types of models and naturally the question of the underlying reasons emerges.

In order to answer this question, we consider single-particle rms radii of the proton orbitals occupied in ${ }^{208} \mathrm{~Pb}$ and ${ }^{220} \mathrm{~Pb}$ as obtained in the calculations without pairing (see Table 1). These nuclei differ by 12 neutrons which completely oc-

Table 1. (Color online) Proton single-particle rms radii $r_{p, i}=\sqrt{\left\langle r^{2}\right\rangle_{i}}$ [in fm] (columns 4 and 5) and single-particle energies $e_{i}$ [in MeV] (columns 2 and 3 ) of the indicated singleparticle $\left(i\right.$-th) in the nuclei ${ }^{208} \mathrm{~Pb}$ and ${ }^{220} \mathrm{~Pb}$. The pairing is neglected in the calculations and they employ NL3* functional. The column 6 shows the change of proton singleparticle radii $\delta r_{p, i}=r_{p, i}\left({ }^{220} \mathrm{~Pb}\right)-r_{p, i}\left({ }^{208} \mathrm{~Pb}\right)$ between the nuclei ${ }^{208} \mathrm{~Pb}$ and ${ }^{220} \mathrm{~Pb}$. Note that the results for the single-particle states with principal quantum number $n=1$ are shown in bold.

\begin{tabular}{|c|c|c|c|c|c|}
\hline \hline subshell & $e_{i}\left({ }^{208} \mathrm{~Pb}\right)$ & $e_{i}\left({ }^{220} \mathrm{~Pb}\right)$ & $r_{p, i}\left({ }^{208} \mathrm{~Pb}\right)$ & $r_{p, i}\left({ }^{220} \mathrm{~Pb}\right)$ & $\delta r_{p, i}$ \\
\hline 1 & 2 & 3 & 4 & 5 & 6 \\
\hline $1 s_{1 / 2}$ & $\mathbf{- 4 8 . 8 8 6}$ & $\mathbf{- 4 8 . 1 5 1}$ & $\mathbf{4 . 0 6 7 7 5 9}$ & $\mathbf{4 . 2 7 7 5 9 5}$ & $\mathbf{0 . 2 0 9 8 3 6}$ \\
$1 p_{3 / 2}$ & $\mathbf{- 4 3 . 1 9 3}$ & $\mathbf{- 4 3 . 1 5 5}$ & $\mathbf{4 . 6 6 7 4 5 4}$ & $\mathbf{4 . 8 8 9 2 0 8}$ & $\mathbf{0 . 2 2 1 7 5 4}$ \\
$1 p_{1 / 2}$ & $\mathbf{- 4 2 . 5 1 0}$ & $\mathbf{- 4 2 . 6 2 0}$ & $\mathbf{4 . 5 7 5 7 3 5}$ & $\mathbf{4 . 8 0 5 2 2 7}$ & $\mathbf{0 . 2 2 9 4 9 2}$ \\
$1 d_{5 / 2}$ & $\mathbf{- 3 6 . 0 9 7}$ & $\mathbf{- 3 6 . 8 5 3}$ & $\mathbf{5 . 1 1 1 5 9}$ & $\mathbf{5 . 3 2 4 1 1 5}$ & $\mathbf{0 . 2 1 2 5 2 5}$ \\
$1 d_{3 / 2}$ & $\mathbf{- 3 4 . 5 3 3}$ & $\mathbf{- 3 5 . 6 3 6}$ & $\mathbf{4 . 9 7 2 5 6 7}$ & $\mathbf{5 . 1 8 7 8 9 7}$ & $\mathbf{0 . 2 1 5 3 3}$ \\
$2 s_{1 / 2}$ & -30.844 & -32.215 & 4.458931 & 4.544538 & 0.085607 \\
$1 f_{7 / 2}$ & $\mathbf{- 2 8 . 0 4 2}$ & $\mathbf{- 2 9 . 5 7 3}$ & $\mathbf{5 . 4 8 7 0 1 9}$ & $\mathbf{5 . 6 8 2 0 7 9}$ & $\mathbf{0 . 1 9 5 0 6}$ \\
$1 f_{5 / 2}$ & $\mathbf{- 2 5 . 2 6 5}$ & $\mathbf{- 2 7 . 3 6 6}$ & $\mathbf{5 . 3 2 5 3 6 4}$ & $\mathbf{5 . 5 1 2 8 4 5}$ & $\mathbf{0 . 1 8 7 4 8 1}$ \\
$2 p_{3 / 2}$ & -20.890 & -22.819 & 5.000532 & 5.032818 & 0.032286 \\
$2 p_{1 / 2}$ & -19.839 & -21.843 & 5.004416 & 5.03297 & 0.028554 \\
$1 g_{9 / 2}$ & $\mathbf{- 1 9 . 3 6 9}$ & $\mathbf{- 2 1 . 5 8 7}$ & $\mathbf{5 . 8 2 5 0 1}$ & $\mathbf{5 . 9 9 8 8 3 9}$ & $\mathbf{0 . 1 7 3 8 2 9}$ \\
$1 g_{7 / 2}$ & $\mathbf{- 1 5 . 1 7 5}$ & $\mathbf{- 1 8 . 1 4 9}$ & $\mathbf{5 . 6 7 3 3 0 5}$ & $\mathbf{5 . 8 2 3 0 3}$ & $\mathbf{0 . 1 4 9 7 2 5}$ \\
$2 d_{5 / 2}$ & -11.154 & -13.413 & 5.53793 & 5.542713 & 0.004783 \\
$1 h_{11 / 2}$ & $\mathbf{- 1 0 . 3 3 5}$ & $\mathbf{- 1 3 . 1 2 0}$ & $\mathbf{6 . 1 4 1 0 4}$ & $\mathbf{6 . 2 9 1 5 7 1}$ & $\mathbf{0 . 1 5 0 5 3 1}$ \\
$2 d_{3 / 2}$ & -9.537 & -11.909 & 5.572499 & 5.577024 & 0.004525 \\
$3 s_{1 / 2}$ & -8.428 & -10.725 & 5.495602 & 5.482959 & -0.012643 \\
\hline \hline
\end{tabular}


cupy the $\nu 1 i_{11 / 2}$ subshell ${ }^{1}$ (see discussion in Ref. [15]). One can $\operatorname{see}^{2}$ that the pull provided by neutrons in this subshell on rms radii of proton single-particle states depends on their principal quantum number $n$. This pull is quantified by $\delta r_{p, i}$ (see Table 1) and it is the largest for the $n=1$ orbitals (especially those located at the bottom of the potential). It is reduced drastically for the $n=2$ and 3 proton orbitals. Note that the proton rms point radius entering into Eq. (1) is given by

$$
\left\langle r^{2}\right\rangle_{p}=\sum_{i}\left(2 j_{i}+1\right) r_{p, i}^{2},
$$

where the sum runs over all occupied spherical subshells and $\left(2 j_{i}+1\right)$ is the degeneracy of the $i$-th subshell. As a result, the differential charge radius between two nuclei (see Eq. (2)) can be rewritten as

$$
\delta\left\langle r^{2}\right\rangle_{p}^{N, N^{\prime}}=\sum_{i}\left(2 j_{i}+1\right)\left(r_{p, i}^{2}(N)-r_{p, i}^{2}\left(N^{\prime}\right)\right) .
$$

One can see that it depends on the pull on the rms radii of all occupied proton subshells provided by the occupation of neutron states. Thus the introduction of the core (as it is done in the spherical shell model) leads to a neglect of the pull provided by the extra neutron(s) on the proton single-particle states forming the core. This introduces uncontrollable errors in the calculations of differential charge radii and thus severely limits the applicability of spherical shell model to the description of this observable.

In a similar fashion, the lack of self-consistency effects is expected to affect the description of differential charge radii in the mic+mac model. This is because the addition of neutron does not affect the proton subsystem in a self-consistent manner on the level of single-particle subshells (as in Table 1). For example, in the Woods-Saxon potential it affect the total radius of nucleus $R$ only via mass dependence $R=1.2 A^{1 / 3}$ : this means that the occupation of the neutron $1 i_{11 / 2}$ and $2 g_{9 / 2}$ subshells in the $N>126 \mathrm{~Pb}$ isotopes will lead to the same differential charge radii contrary to the results of self-consistent calculations (see Refs. $[4,5,24,37]$.) Moreover, there is a lack of self-consistency in the definition of the radial properties of the density distributions in the macroscopic (liquid drop) and microscopic (single-particle potential) parts of the mic+mac model. To our knowledge, this aspect of the problem has not been studied in details. However, the physical observables similar to $\delta\left\langle r^{2}\right\rangle_{p}^{N, N^{\prime}}$, namely, relative charge quadrupole moments of superdeformed bands are affected by the lack of selfconsistency between microscopic and macroscopic parts [43].

\footnotetext{
${ }^{1}$ We consider a full neutron subshell in order to magnify the impact of the occupation of neutron states on the proton single-particle rms radii.

${ }^{2}$ Similar impact of the occupation of the $1 i_{11 / 2}$ neutron subshell on charge radii of proton subshells is seen also in the Skyrme DFT calculations of Ref. [41].
} 
Global Performance of CDFT in Description of Charge Radii and Related Indicators

\section{Conclusions}

Recent applications of covariant density functional theory to the analysis and description of charge radii and related indicators have been reviewed. It covers global accuracy of the description of experimental absolute and differential charge radii and the evaluation of related theoretical statistical and systematic uncertainties. This theory provides reasonably good description of these physical observables already at the mean field level. The discrepancies between theory and experiment have two sources: the deficiencies in the description of the energies of the single-particle states and the neglect of the correlations beyond mean field. For example, the former affects the reproduction of the evolution of differential charge radii in isotopic chains which cross the $N=28$ and $N=82$ neutron shell closures. The later shows itself in two areas: (i) in the description of charge radii in shape coexistent nuclei or in the nuclei with soft potential energy surfaces [accurate description of such systems requires the methods such as generator coordinate method or 5-dimensional collective Hamiltonian] and (ii) in the description of odd-even staggering in charge radii since the energies and the wave functions of one-quasiparticle states in odd- $A$ nuclei are affected [strongly in spherical nuclei] by particle-vibration coupling.

In addition, new calculations reveal that differential charge radii in deformed actinides and light superheavy nuclei are well described in the CDFT calculations. However, it is important to take into account octupole deformation in low- $N$ $\mathrm{Th}$ and $\mathrm{U}$ nuclei. Finally, we analyzed the importance of self-consistency effects on the accurate description of differential charge. This analysis reveals the challenging situation. The CDFT as well as the Skyrme and Gogny DFTs take these effects into account on the level of single-particle states but suffer from some deficiencies in the description of their energies. In contrast, the models which are specifically fitted for a very accurate description of the single-particle energies suffer from the neglect of these self-consistency effects either due to introduction of the closed core (as in the most of spherical shell models) or due to the split of the model space into macroscopic and microscopic part (as in the mic+mac models).

\section{Acknowledgements}

This material is based upon work supported by the U.S. Department of Energy, Office of Science, Office of Nuclear Physics under Award No. DESC0013037. PR acknowledges partial support from the Deutsche Forschungsgemeinschaft (DFG, German Research Foundation) under Germany Excellence Strategy EXC-2094-390783311, ORIGINS. 


\section{References}

[1] I. Angeli, K.P. Marinova (2013) At. Data Nucl. Data Tables 9969.

[2] P. Campbell, I. Moore, M. Pearson (2016) Prog. Part. Nucl. Phys. 86127.

[3] D. Vretenar, A.V. Afanasjev, G.A. Lalazissis, P. Ring (2005) Phys. Rep. 409101.

[4] M.M. Sharma, G.A. Lalazissis, P. Ring (1993) Phys. Lett. B 3179.

[5] P.-G. Reinhard, H. Flocard (1995) Nucl. Phys. A 584467.

[6] Y. Tian, Z.Y. Ma, P. Ring (2009) Phys. Lett. B 67644.

[7] S.E. Agbemava, A.V. Afanasjev, D. Ray, P. Ring (2014) Phys. Rev. C 89054320.

[8] G.A. Lalazissis, S. Karatzikos, R. Fossion, D. Peña Arteaga, A.V. Afanasjev, P. Ring (2009) Phys. Lett. B 67136.

[9] G.A. Lalazissis, T. Nikšić, D. Vretenar, P. Ring (2005) Phys. Rev. C 71024312.

[10] X. Roca-Maza, X. Viñas, M. Centelles, P. Ring, P. Schuck (2011) Phys. Rev. C 84 055309.

[11] T. Nikšić, D. Vretenar, P. Ring (2008) Phys. Rev. C 78034318.

[12] P.W. Zhao, Z.P. Li, J.M. Yao, J. Meng (2010) Phys. Rev. C 82054319.

[13] S.E. Agbemava, A.V. Afanasjev, A. Taninah (2019) Phys. Rev. C 99014318.

[14] A.V. Afanasjev, S.E. Agbemava (2016) Phys. Rev. C 93054310.

[15] U.C. Perera, A.V. Afanasjev, P. Ring (2021) Phys. Rev. C, in press; see also nuclear theory arXiv:2108.02245.

[16] S. Frauendorf, V.V. Pashkevich (1975) Phys. Lett. B 55365.

[17] J.-P. Delaroche, M. Girod, J. Libert, H. Goutte, S. Hilaire, S. Peru, N. Pillet, G.F. Bertsch (2010) Phys. Rev. C 81014303.

[18] E.W. Otten (1989) Nuclear radii and moments of unstable isotopes. In: "Treatise on Heavy Ion Science: Volume 8: Nuclei Far From Stability”, edited by D.A. Bromley (Springer, Boston, MA), pp. 517-638.

[19] S. Sels et al. (2019) Phys. Rev. C 99044306.

[20] M. Bender, P.-H. Heenen, P.-G. Reinhard (2003) Rev. Mod. Phys. 75121.

[21] J. Boguta, R. Bodmer (1971) Nucl. Phys. A 292413.

[22] J. Dobaczewski, W. Nazarewicz, P.-G. Reinhard (2014) J. Phys. G 41074001.

[23] Y. Gao, J. Dobaczewski, M. Kortelainen, J. Toivanen, D. Tarpanov (2013) Phys. Rev. C 87034324.

[24] T.D. Goodacre et al. (2021) Phys. Rev. C 104054322.

[25] R.F. Garcia-Ruiz, A. Vernon (2020) Eur. Phys. J. A 56136.

[26] S.E. Agbemava, A.V. Afanasjev, P. Ring (2016) Phys. Rev. C 93044303.

[27] A. Voss, V. Sonnenschein, P. Campbell, B. Cheal, T. Kron, I.D. Moore, I. Pohjalainen, S. Raeder, N. Trautmann, K. Wendt (2017) Phys. Rev. A 95032506.

[28] S. Raeder et al. (2018) Phys. Rev. Lett. 120232503 (2018).

[29] Saleh O. Allehabi, V.A. Dzuba, V.V. Flambaum, A.V. Afanasjev, S.E. Agbemava (2020) Phys. Rev. C 102024326.

[30] Yunchen Cao, S.E. Agbemava, A.V. Afanasjev, W. Nazarewicz, E. Olsen (2020) Phys. Rev. C 102024311.

[31] A.V. Afanasjev, S. Shawaqfeh (2011) Phys. Lett. B 706177.

[32] L. Bonneau, P. Quentin, P. Möller (2007) Phys. Rev. C 76024320. 
Global Performance of CDFT in Description of Charge Radii and Related Indicators

[33] J. Dobaczewski, A.V. Afanasjev, M. Bender, L.M. Robledo, Yue Shi (2015) Nucl. Phys. A 944388.

[34] S.A. Fayans, S.V. Tolokonnikov, E.L. Trykov, D. Zawischa (1994) Phys. Lett. B 338 1.

[35] S.A. Fayans, S.V. Tolokonnikov, E.L. Trykov, D. Zawischa (2000) Nucl. Phys. A 67649.

[36] P.-G. Reinhard, W. Nazarewicz (2017) Phys. Rev. C 95064328.

[37] T.D. Goodacre et al. (2021) Phys. Rev. Let. 126032502.

[38] E.V. Litvinova, A.V. Afanasjev (2011) Phys. Rev. C 84014305.

[39] A.V. Afanasjev, E. Litvinova (2015) Phys. Rev. C 92044317.

[40] E. Litvinova, P. Ring (2006) Phys. Rev. C 73044328.

[41] P.M. Goddard, P.D. Stevensson, A. Rios (2013) Phys. Rev. Lett. 110032503.

[42] G. Coló, H. Sagawa, P.F. Bortignon (2010) Phys. Rev. C 82064307.

[43] L.B. Karlsson, I. Ragnarsson, S. Åberg (1998) Nucl. Phys. A 639654. 